Portland State University

PDXScholar

6-10-2009

\title{
Monte Carlo Green's Function Formalism for the Propagation of Partially Coherent Light
}

\author{
Scott A. Prahl \\ David G. Fischer \\ Donald D. Duncan \\ Portland State University
}

Follow this and additional works at: https://pdxscholar.library.pdx.edu/ece_fac

Part of the Electrical and Computer Engineering Commons

Let us know how access to this document benefits you.

\section{Citation Details}

Scott A. Prahl, David G. Fischer, and Donald D. Duncan, "Monte Carlo Green's function formalism for the propagation of partially coherent light," J. Opt. Soc. Am. A 26, 1533-1543 (2009)

This Article is brought to you for free and open access. It has been accepted for inclusion in Electrical and Computer Engineering Faculty Publications and Presentations by an authorized administrator of PDXScholar. Please contact us if we can make this document more accessible: pdxscholar@pdx.edu. 


\title{
Monte Carlo Green's function formalism for the propagation of partially coherent light
}

\author{
Scott A. Prahl, ${ }^{1, *}$ David G. Fischer, ${ }^{2}$ and Donald D. Duncan ${ }^{3}$ \\ ${ }^{1}$ Oregon Medical Laser Center, Providence St. Vincent Medical Center, 9205 SW Barnes Road, Portland, \\ Oregon 97225, USA \\ ${ }^{2}$ NASA Glenn Research Center, 21000 Brookpark Road, Cleveland, Ohio 44135, USA \\ ${ }^{3}$ Division of Biomedical Engineering, Oregon Health \& Science University, 3303 SW Bond Avenue, Portland, \\ Oregon 97239, USA \\ *Corresponding author: prahl@bme.ogi.edu
}

Received February 27, 2009; revised May 6, 2009; accepted May 8, 2009; posted May 11, 2009 (Doc. ID 108137); published June 10, 2009

\begin{abstract}
We present a Monte Carlo-derived Green's function for the propagation of partially spatially coherent fields. This Green's function, which is derived by sampling Huygens-Fresnel wavelets, can be used to propagate fields through an optical system and to compute first- and second-order field statistics directly. The concept is illustrated for a cylindrical f/1 imaging system. A Gaussian copula is used to synthesize realizations of a Gaussian Schell-model field in the pupil plane. Physical optics and Monte Carlo predictions are made for the first- and second-order statistics of the field in the vicinity of the focal plane for a variety of source coherence conditions. Excellent agreement between the physical optics and Monte Carlo predictions is demonstrated in all cases. This formalism can be generally employed to treat the interaction of partially coherent fields with diffracting structures. (C) 2009 Optical Society of America
\end{abstract}

OCIS codes: $030.5620,030.1670,030.6600,110.4980,170.3660$.

\section{INTRODUCTION}

The propagation of light in complex, strongly scattering random media is an important problem in diagnostic imaging and remote sensing [1-5]. Specific applications include laser communication through the atmosphere [1,2], imaging in biological media and (underwater) littoral environments [3-5], and imaging in extreme environments such as turbulent combustion.

Often, the scattering experienced on propagation is a nuisance. Such is the case in optical telecommunications within the atmosphere [1,2]. In other cases, the effects of scattering are useful because they convey information about the medium itself; the laser Doppler technique for measuring velocity is a good example [6]. Many practical situations involve a combination of these two; an object that one seeks to observe is embedded in a scattering medium that frustrates the observation. Confocal microscopy is a good example of such a case [7]. Here, the source fields are focused to a plane within the medium that one wishes to image. In proceeding from the pupil to the focal spot, however, the fields are subject to scatter that degrades the quality of focus. Moreover, the light backscattered from the focal spot is subject to additional scatter in its path back to the pupil.

Because of the complexity of the interaction in strongly scattering media (such as biological tissue), physical optics (PO) methods of analysis are infeasible. In such cases, researchers have relied almost exclusively upon Monte Carlo (MC) methods based on radiative transfer theory [8-10]. Such methods employ an effective-medium concept that views the medium as having certain scatter and absorption characteristics that are otherwise uniformly distributed. In other words, the medium is viewed as being homogeneous. Objects embedded within the medium (about which information may be desired) are viewed as having different scatter and absorption properties, but are otherwise assumed homogeneous as well. While this has been successful in mimicking empirical results, the method conveys no information about the actual lightmatter interaction.

Traditional MC methods allow only single-point characterizations of the observed field (intensity, polarization), and assume that there is no correlated structure at the scale of the wavelength within the propagation medium. In general, a more complete statistical characterization of the field (second-order moments and higher) is needed. This is accomplished within the framework of optical coherence theory [11]. Accounting for coherence effects is critical, as it is the spatial coherence of the field that affects the quality of focus and ultimately imaging performance (in confocal microscopy, for instance). In addition, it has been shown that spatial coherence changes, even on propagation through free space.

Ideally, then, a complete and efficient treatment of the propagation of light in random media would involve the application of MC methods (or ray tracing in general) within the framework of optical coherence theory. There have been several recent efforts along these lines, from both analytical and numerical perspectives. Zysk et al. [12] employed an eikonal formalism for propagating the cross-spectral density of a special class of partially coherent sources within the geometrical optics regime. Petruccelli and Alonso [13] conducted a general analytical study of the propagation of the cross-spectral density using ray 
tracing. Douglas et al. [14] developed a ray-based simulation for an optical interferometer that accounted for the evolution of spatial coherence from an extended source, but used systematic angular ray sampling and neglected diffraction. A paraxial ray-based simulation was also developed by Riechert et al. [15], but they too used a systematic sampling algorithm, neglected diffraction, and did not specifically treat the case of arbitrary coherence.

In a recent paper [16], we presented a Monte Carlo (MC) method for propagating partially coherent field realizations which integrates the physical optics (PO) description of light with ray-based propagation techniques. The combination of the two methodologies preserves the wave nature of light, which is essential for describing coherence phenomena, while exploiting the computational simplicity and robustness of MC techniques [10-12,17], allowing one to model complex phenomena that cannot be treated by either approach individually.

In this paper, we present an extension that involves MC computation of the Green's function for the overall system. The Green's function can be used to propagate individual realizations and compute arbitrary moments of the propagated field, as in [16]. Unlike the previous technique, however, it can also be used to propagate the crossspectral density (a second-order moment) directly, at a substantial computational savings. We illustrate our Green's function technique using an f/1 imaging system. It should be noted, however, that our technique can be applied to any complex medium for which the light-matter interaction can be modeled as either the absorption or redirection (i.e., scattering) of a light ray. The case of propagation through a multiple-scattering medium will be treated in a future paper.

\section{GREEN'S FUNCTION FORMULATION}

The optical configuration that we address herein is that of a two-dimensional f/1 imaging system, although the formalism is generally applicable. We assume that the field within the pupil of the system is partially coherent. We discuss the analytic theory describing such a configuration and present a Green's function implementation of the calculations that are subsequently carried out by numerical integration for the $\mathrm{PO}$ case and by $\mathrm{MC}$ ray trace.

Consider a focused cylindrical wave of frequency $\omega$ that is exiting an aperture of width $2 a$ in a plane screen (see Fig. 1). The axial coordinate is $z, y$ is parallel to the long axis of the cylindrical wave, and $x$ is across the slit. The origin $O$ of the coordinate system coincides with the geometrical focus. The amplitude of the field in the aperture is $U^{(0)}\left(\mathbf{r}^{\prime}, \omega\right), \mathbf{r}^{\prime}$ being the position vector of typical point $Q\left(\mathbf{r}^{\prime}\right)$. The field at a point $P(\mathbf{r})$ in the focal region is, according to the Huygens-Fresnel principle, expressed in two dimensions as [18]

$$
U(\mathbf{r}, \omega)=\frac{i \pi}{\lambda} \int_{-a}^{a} U^{(0)}\left(\mathbf{r}^{\prime}, \omega\right) \exp \left(-i k \sqrt{f^{2}+x^{\prime 2}}\right) \frac{z}{s} H_{1}^{(1)}(k s) \mathrm{d} x^{\prime},
$$

where $\lambda$ is the wavelength, $k=2 \pi / \lambda$ is the wavenumber, $f$ is the focal distance, $s=\left|\mathbf{r}-\mathbf{r}^{\prime}\right|$ denotes the distance $Q P$, and we have suppressed the periodic time-dependent fac-

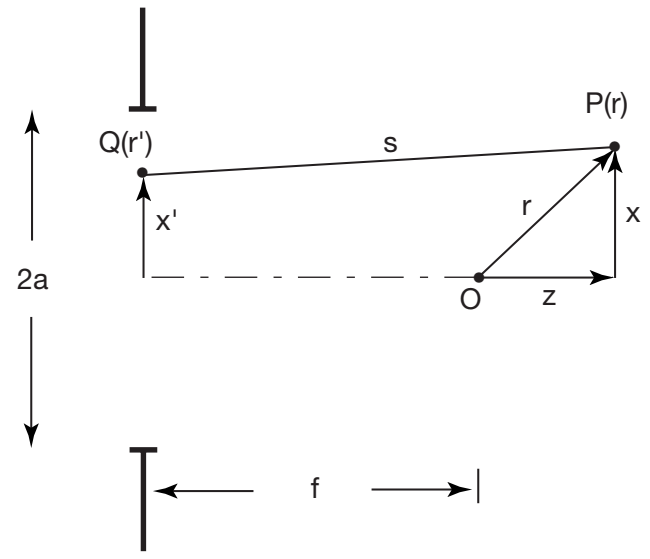

Fig. 1. Illustration of geometrical configuration.

tor $\exp (-i \omega t)$. Alternatively, the field can be expressed in the form

$$
U(\mathbf{r}, \omega)=\int_{-\infty}^{\infty} U^{(0)}\left(\mathbf{r}^{\prime}, \omega\right) G\left(\mathbf{r}, \mathbf{r}^{\prime}\right) \mathrm{d} x^{\prime}
$$

where

$$
G\left(\mathbf{r}, \mathbf{r}^{\prime}\right)=\left\{\begin{array}{ll}
\frac{i \pi}{\lambda} \exp \left(-i k \sqrt{f^{2}+x^{\prime 2}}\right) \underset{s}{z} H_{1}^{(1)}(k s), & \left|x^{\prime}\right|<a \\
0, & \text { else }
\end{array} .\right.
$$

$G\left(\mathbf{r}, \mathbf{r}^{\prime}\right)$ is the Green's function of the system.

A partially coherent wave field is characterized by its statistical moments. One such (second-order) moment is the cross-spectral density function, which describes firstorder correlations in the field ([11], Sect. 2.4.4) and is defined at the points $P\left(\mathbf{r}_{1}\right)$ and $P\left(\mathbf{r}_{2}\right)$ by

$$
W\left(\mathbf{r}_{1}, \mathbf{r}_{2}, \omega\right)=\left\langle U^{*}\left(\mathbf{r}_{1}, \omega\right) U\left(\mathbf{r}_{2}, \omega\right)\right\rangle .
$$

Here the angle brackets denote the average, taken over a statistical ensemble of monochromatic, i.e., time coherent realizations $U(\mathbf{r}, \omega) \exp (-i \omega t)$ ([11], Sect. 4.7), and the asterisk denotes the complex conjugate. One can also define the spectral density and spectral degree of coherence, respectively, as

$$
\begin{aligned}
S(\mathbf{r}) & =W(\mathbf{r}, \mathbf{r}), \\
\mu\left(\mathbf{r}_{1}, \mathbf{r}_{2}\right) & =W\left(\mathbf{r}_{1}, \mathbf{r}_{2}\right) /\left[S\left(\mathbf{r}_{1}\right) S\left(\mathbf{r}_{2}\right)\right]^{1 / 2},
\end{aligned}
$$

Substituting Eq. (2) into Eq. (4), we find that the crossspectral density of the observation plane field has the form

$$
\begin{aligned}
W\left(\mathbf{r}_{1}, \mathbf{r}_{2}, \omega\right)= & \int_{-\infty}^{\infty} \int_{-\infty}^{\infty} W^{(0)}\left(\mathbf{r}_{1}^{\prime}, \mathbf{r}_{2}^{\prime}, \omega\right) \\
& \times G^{*}\left(\mathbf{r}_{1}, \mathbf{r}_{1}^{\prime}\right) G\left(\mathbf{r}_{2}, \mathbf{r}_{2}^{\prime}\right) \mathrm{d} x_{1}^{\prime} \mathrm{d} x_{2}^{\prime},
\end{aligned}
$$

where $W^{(0)}\left(\mathbf{r}^{\prime}{ }_{1}, \mathbf{r}^{\prime}{ }_{2}, \omega\right)$ is the cross-spectral density of the field in the aperture. For the source fields used herein, we assumed a Gaussian Schell-model with uniform amplitude ([11], Sec. 5.3), i.e., 


$$
W^{(0)}\left(\mathbf{r}^{\prime}, \mathbf{r}^{\prime \prime}\right)=W^{(0)}\left(x^{\prime}, x^{\prime \prime}\right)=\exp \left[-\frac{\left(x^{\prime}-x^{\prime \prime}\right)^{2}}{2 \sigma_{g}^{2}}\right],
$$

where $x^{\prime}$ and $x^{\prime \prime}$ are points on the wavefront in the aperture and $\sigma_{g}$ is a positive constant that is a measure of the effective spectral coherence length of the field in the aperture.

Recently [16], we presented a method for propagating individual field realizations using Eq. (1). That method involved generating input (or aperture) field realizations corresponding to a prescribed cross-spectral density using a Gaussian copula algorithm $[19,20]$, propagating each field realization by MC sampling of Eq. (1) and averaging over the field ensemble at the output plane [in accordance with Eq. (4)] to compute the cross-spectral density. The computational demands imposed by this method would make it appear that generalization to higher-dimensional problems (three-dimensional waves, polarization, multiple scattering) may be computationally infeasible.

However, it is clear from Eq. (2) that a more expeditious procedure would be to precompute the system Green's function by MC sampling of Eq. (3). This Green's function then could be used to propagate each field realization. Furthermore, if one is interested only in secondorder statistics (i.e., the cross-spectral density), the precomputed Green's function can be used in Eq. (6) directly. Since the waves are cylindrical, the pupil plane fields may be represented in terms of an $N^{\prime} \times 1$ vector, the focal plane fields by an $N \times 1$ vector, and the Green's function by an $N \times N^{\prime}$ matrix. Propagation of the individual field realizations then amounts to a series of matrix multiplications:

$$
U=G U^{(0)} .
$$

\section{NUMERICAL IMPLEMENTATION}

In this section we describe the MC procedure for computing the system Green's function, the copula method for generating field realizations conforming to the GaussianSchell model, direct calculation of the first- and secondorder statistics of the field from the Green's function, and the corrections needed in the PO calculation to account for the finite size of detector elements.

\section{A. Monte Carlo Ray Tracing}

The Green matrix, $G$, is generated by tracing rays from the pupil to the detector; the $G_{i j}$ element is the coherent sum of all fields starting at the $j$ th source element and reaching the $i$ th detector element. Equal numbers of rays are launched from each source element. The initial field associated with each ray has unit amplitude and zero phase. The starting location of each ray is randomly chosen across each source element (simply launching from the center would create a diffraction grating).

The initial direction of the ray is chosen to randomly sample the emerging Huygens-Fresnel wavelet. If $\xi$ is a uniform random deviate over the interval $[0,1]$, then the initial angle might be $\theta=2 \pi \xi$. Such a launch angle would obviously be inefficient because most rays would not reach the detector. Instead, if the minimum and maxi- mum angles $\theta_{\min }$ and $\theta_{\max }$ between the current location and the next aperture are calculated, then the launch angle might be

$$
\theta=\theta_{\min }+\xi\left(\theta_{\max }-\theta_{\min }\right)
$$

This ensures that every ray passes through all apertures, but also contains a subtle sampling bias because the number of rays/radian varies with the subtended angle $\theta_{\max }$ $-\theta_{\min }$. This is corrected by multiplying the field amplitude associated with each ray by the subtended angle. No other corrections are needed. For example, the typical $1 / \sqrt{r}$ falloff in a cylindrical field is implicitly included because the number of rays reaching a particular location will diminish with distance. Obliquity factors are also not needed.

The field associated with a ray that has traveled a distance $d$ must have the phase of its field increased by $k d$. If the field passes through a lens having a focal length of $f$, then the phase is decreased by $k \sqrt{x^{2}+f^{2}}$, where $x$ is the intersection point of the ray with the lens.

Slightly different observation plane sampling is used depending on whether axial or transverse field information is being collected. The transverse case uses the point of intersection in the focal plane to determine the proper detector element to increment by the ray's field. The axial case uses $N$ detector elements distributed at uniform intervals near the focal point. Each axial element is then treated as if it were a transverse detector with a single element.

The modulus and phase of the PO and MC Green's functions are displayed, respectively, in Figs. 2 and 3. Each display corresponds to a matrix with $N^{\prime}=51$ source points (columns) and $N=201$ focal plane points (rows). In this example, the extent of the focal plane dimension is limited to $\pm 2.5 \lambda$. For the MC Green's function, a total of $10^{8}$ rays are traced. For the PO Green's function, a numerical evaluation of Eq. (3) is used. For purposes of comparison in Figs. 2 and 3, a measurement function (see Subsection 3.D) is applied to the PO Green's function.

\section{B. Generation of Partially Coherent Field Realizations}

Using the Gaussian copula algorithm $[16,20]$ we generate a series of zero mean circularly complex Gaussian field realizations having prescribed amplitude and correlation properties. We briefly review this algorithm.

Consider two uniformly distributed, statistically independent random variables (RVs), $X_{1}$ and $X_{2}$. From this pair, the Box-Muller transformation [21] produces a new pair of RVs,

$$
\begin{aligned}
& Y_{1}=\sqrt{-2 \ln X_{1}} \cos \left(2 \pi X_{2}\right), \\
& Y_{2}=\sqrt{-2 \ln X_{1}} \sin \left(2 \pi X_{2}\right),
\end{aligned}
$$

that are jointly Normal with zero correlation coefficient, $N(\mu, \sigma, r)=N(0,1,0)$. Next, making use of the scaling and rotation operations, 

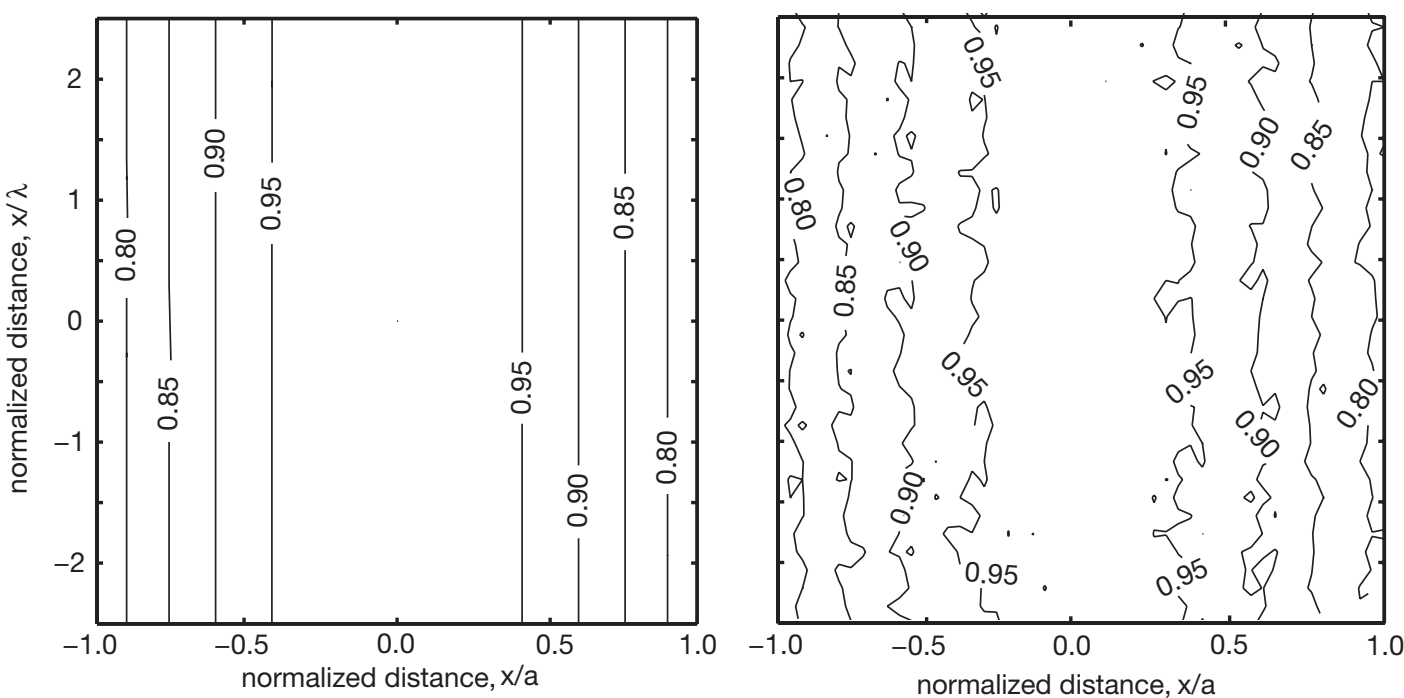

Fig. 2. Normalized modulus of Green's function from numerical evaluation of Eq. (3) (left) and of MC ray trace (right). For the MC Green's function, a total of $10^{8}$ rays were traced. For purposes of this comparison, a measurement function (see Subsection 3.D) was applied to the PO Green's function.

$$
\left[\begin{array}{l}
Z_{1} \\
Z_{2}
\end{array}\right]=\frac{1}{\sqrt{2}}\left[\begin{array}{rr}
1 & -1 \\
1 & 1
\end{array}\right]\left[\begin{array}{cc}
\sqrt{1+r} & 0 \\
0 & \sqrt{1-r}
\end{array}\right]\left[\begin{array}{l}
Y_{1} \\
Y_{2}
\end{array}\right]
$$

we find that the RV's, $Z_{1}$ and $Z_{2}$, are correlated, bivariate Normal, $N(0,1, r)$. The Box-Muller transformation and the scaling and rotation constitute the copula that links the marginal distributions on $X_{1}$ and $X_{2}$ into the bivariate distribution on $Z_{1}$ and $Z_{2}$.

Spatially band limited random field realizations $[20,22]$ are synthesized using the following procedure: Create an $L \times L$ element matrix of zeros and fill the central circular region of diameter $K$ elements with complex numbers of unit amplitudes and phases that are effectively uniformly distributed over $(0,2 \pi)$. Upon Fourier transforming the $L \times L$ array, one obtains a synthetic field pattern having a Rayleigh probability distribution. The ratio of $L$ to $K$ sets the length of the spatial autocorrelation of the field real-

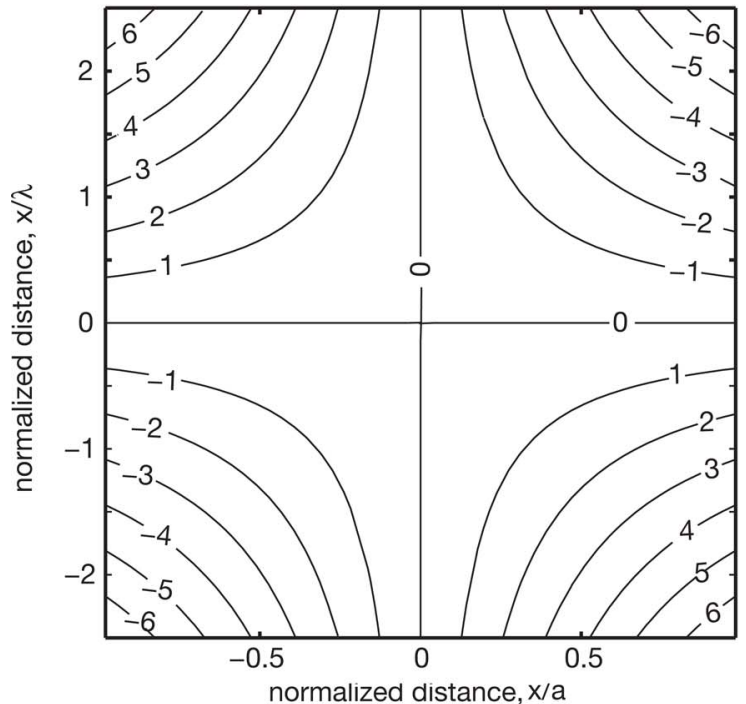

ization. For example if $L=2 K$, the Nyquist criterion is met and the length of the autocorrelation is of the order of two pixels. Use of the phases $\phi_{1}=2 \pi m z_{1}, \phi_{2}=2 \pi m z_{2}$ in this procedure, where $z_{1}$ and $z_{2}$ are effectively uniformly distributed samples (see Appendix A for a discussion of this detail) as in the preceding discussion and $m$ is a real constant, produces a pair of correlated field realizations. The actual correlation between these two realizations from the complex Gaussian moment theorem [23] is given by

$$
\mu=\exp \left(-\frac{1}{2} \sigma_{\Delta \phi}^{2}\right),
$$

where $\sigma_{\Delta \phi}^{2}=\operatorname{var}\left(\phi_{1}-\phi_{2}\right)$ is the variance of the phase difference. Using a sequence of $N^{\prime}$ correlation values between $r=1$ and $r=-1$ [see Eq. (11)] produces a $K \times K \times N^{\prime}$ cube of field realizations that slowly decorrelate between the first

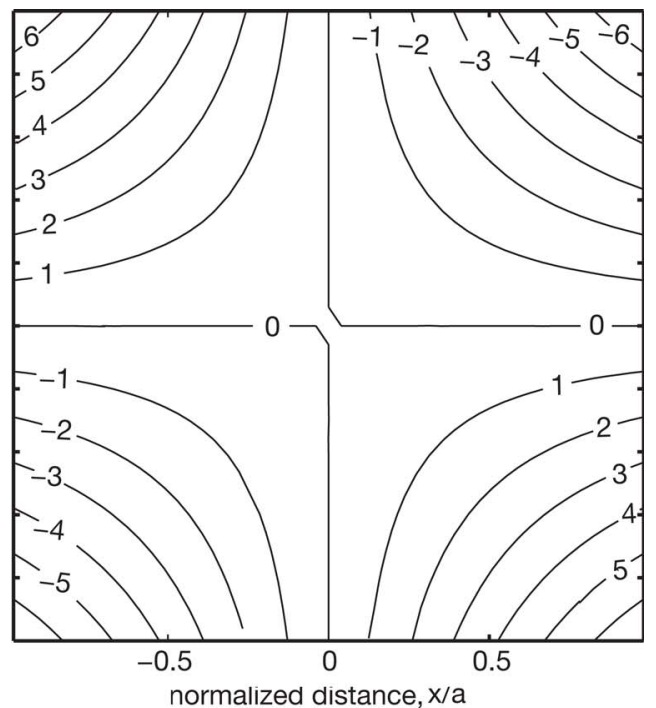

Fig. 3. Phase in radians of Green's function from numerical evaluation of Eq. (9) (left) and of MC ray trace (right). These displays are relative to the zero phase value at $(0,0)$. For purposes of this comparison, a measurement function (see Subsection 3.D) was applied to the PO Green's function. 

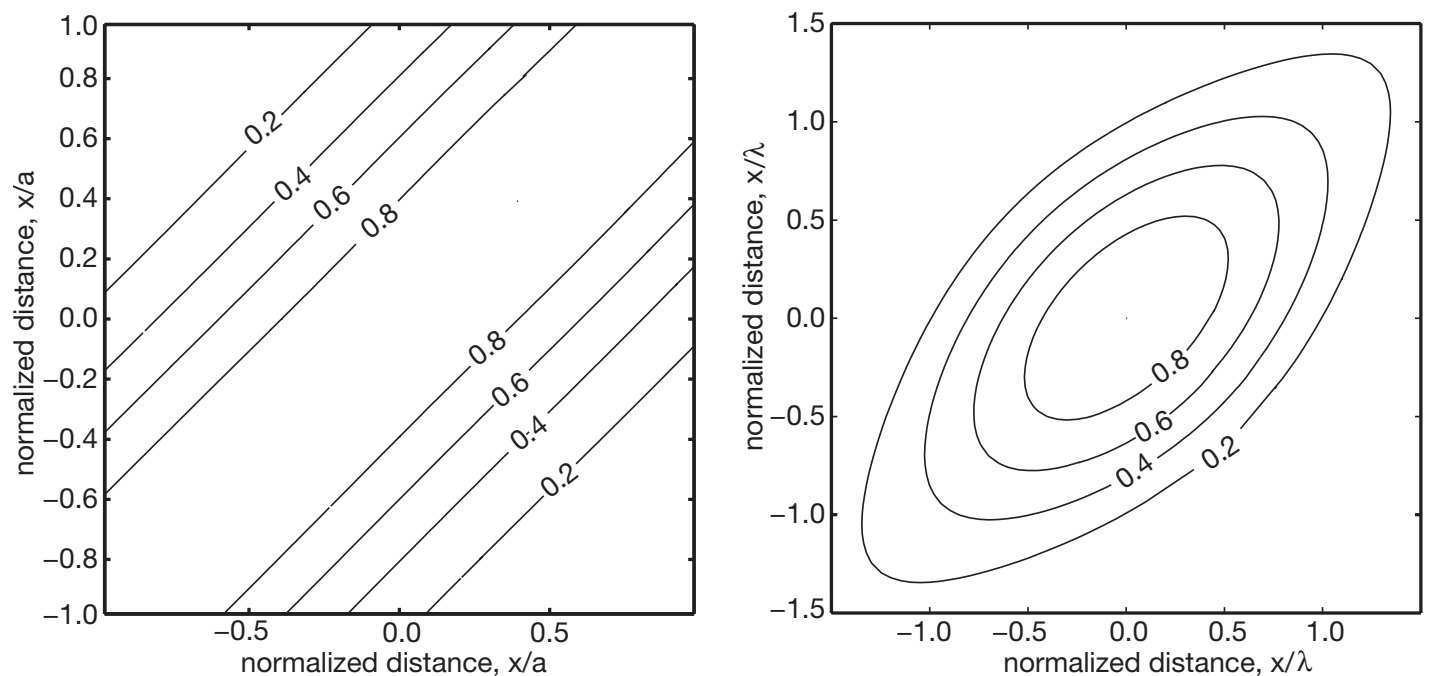

Fig. 4. Normalized moduli of cross-spectral density matrices for source $\sigma_{g} / a=0.6$ (left) and focal plane (right).

$K \times K$ element and the last $K \times K$ element. Elements of this field cube can be interpreted [16] as $K^{2}$ onedimensional ensemble members, each of length $N^{\prime}$.

Following this procedure, ensembles of line source realizations having prescribed spatial correlation properties are synthesized. These ensembles contain $(256)^{2}$ realizations (each with 51 elements) with correlation lengths of $\sigma_{g} / a=2.0,1.0,0.6,0.4,0.2$, and 0.1 .

\section{Propagation of the Statistics}

As suggested in Section 2, the Green's function approach could be used to propagate the individual field realizations via a series of simple matrix multiplications. A further simplification is possible through consideration of the cross-spectral density matrix of the observation fields.

To begin, we consider the vector matrix expression of Eq. (8), $U=G U^{(0)}$, where $U$ and $U^{(0)}$ are now assumed to be $N \times N_{f}$ matrices and $N_{f}$ is the number of realizations of the field ensemble. The matrix analog of the relationship shown in Eq. (6) is given by

$$
\begin{aligned}
W & =\left(1 / N_{f}\right) U U^{\dagger}=\left(1 / N_{f}\right)\left(G U^{(0)}\right)\left(G U^{(0)}\right)^{\dagger} \\
& =G\left[\left(1 / N_{f}\right) U^{(0)} U^{(0) \dagger}\right] G^{\dagger}=G W^{(0)} G^{\dagger},
\end{aligned}
$$

where the dagger denotes the conjugate transpose. Note that the matrices $W^{(0)}$ and $W$ are specific expressions of the general cross-spectral densities $W^{(0)}\left(\mathbf{r}_{i}, \mathbf{r}_{j}\right)$ and $W\left(\mathbf{r}_{i}, \mathbf{r}_{j}\right)$. It is seen that the cross-spectral density of the observation plane field is given by simple left and right Green's matrix multiplications of the source crossspectral density matrix.

In Fig. 4, we illustrate the source and focal plane crossspectral density matrices for the case $\sigma_{g} / a=0.6$. These matrices incorporate the complete first- and second-order statistics of the field. Specifically, the spectral densities are the diagonals of the respective matrices, and the spectral degree of coherence in symmetric form $\mu(-x, 0,0 ; x, 0,0)$ are the cross-diagonals (from northwest to southeast) divided by the spectral densities. Note that these matrices are displayed in Cartesian rather than standard matrix format. The asymmetric form of the spectral degree of coherence, $\mu(0,0,0 ; x, 0,0)$, is simply the central row or column of the cross-spectral density matrix divided by the square root of the product of the spectral density trace and the spectral density at the center of the matrix $\sqrt{S(x, 0,0) S(0)}$. The corresponding axial cross-spectral density matrix in the vicinity of the focal plane is shown in Fig. 5.

\section{Measurement Function}

The MC method intrinsically predicts the output of a physical detector, whereas the PO method does not. In order to directly compare the two, the PO results must be related to measurable quantities. This is accomplished by operating on the native $\mathrm{PO}$ results with a so-called measurement function. This measurement function explicitly accounts for the local propagation direction of the field with respect to the detector surface normal so that the relevant physical quantities (e.g., the irradiance) are properly calculated. Application of the measurement function involves calculating a flux vector from which the de-

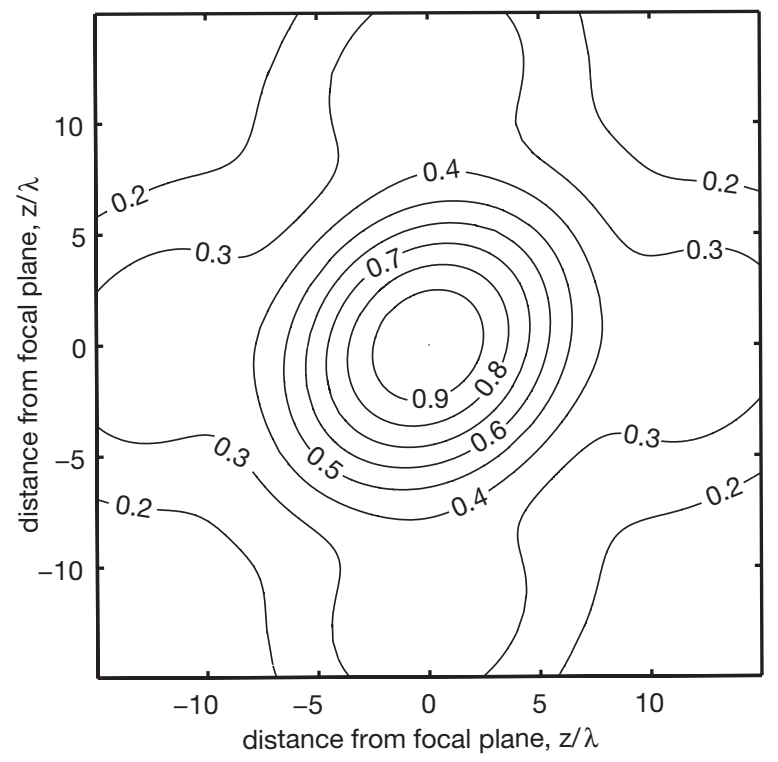

Fig. 5. Axial cross-spectral density matrix in the vicinity of the focal plane for the case $\sigma_{g} / a=0.6$. 
tected intensity and cross-spectral density are determined. This parallels electromagnetic theory, where the Poynting vector (a flux vector calculated from the electric and magnetic fields) is used to determine energy absorption. In the deterministic case, the flux density vector corresponding to the field $U$ is given by

$$
\mathbf{F}(\mathbf{r})=1 / 2 i k\left[U(\mathbf{r}) \nabla U^{*}(\mathbf{r})-U^{*}(\mathbf{r}) \nabla U(\mathbf{r})\right],
$$

where $\nabla$ is the gradient operator. The measurable, in this case the irradiance, is then calculated by taking the scalar dot product of the flux density with the normal to the detector surface. For random fields, the development proceeds along the same lines. One can define a crossspectral flux density vector by [13]

$$
\begin{aligned}
\mathbf{F}_{c}\left(\mathbf{r}_{1}, \mathbf{r}_{2}\right) & =1 / 2 i k\left\langle U^{*}\left(\mathbf{r}_{1}\right) \nabla_{2} U\left(\mathbf{r}_{2}\right)-U\left(\mathbf{r}_{2}\right) \nabla_{1} U^{*}\left(\mathbf{r}_{1}\right)\right\rangle \\
& =1 / 2 i k\left(\nabla_{2}-\nabla_{1}\right) W\left(\mathbf{r}_{1}, \mathbf{r}_{2}\right) .
\end{aligned}
$$
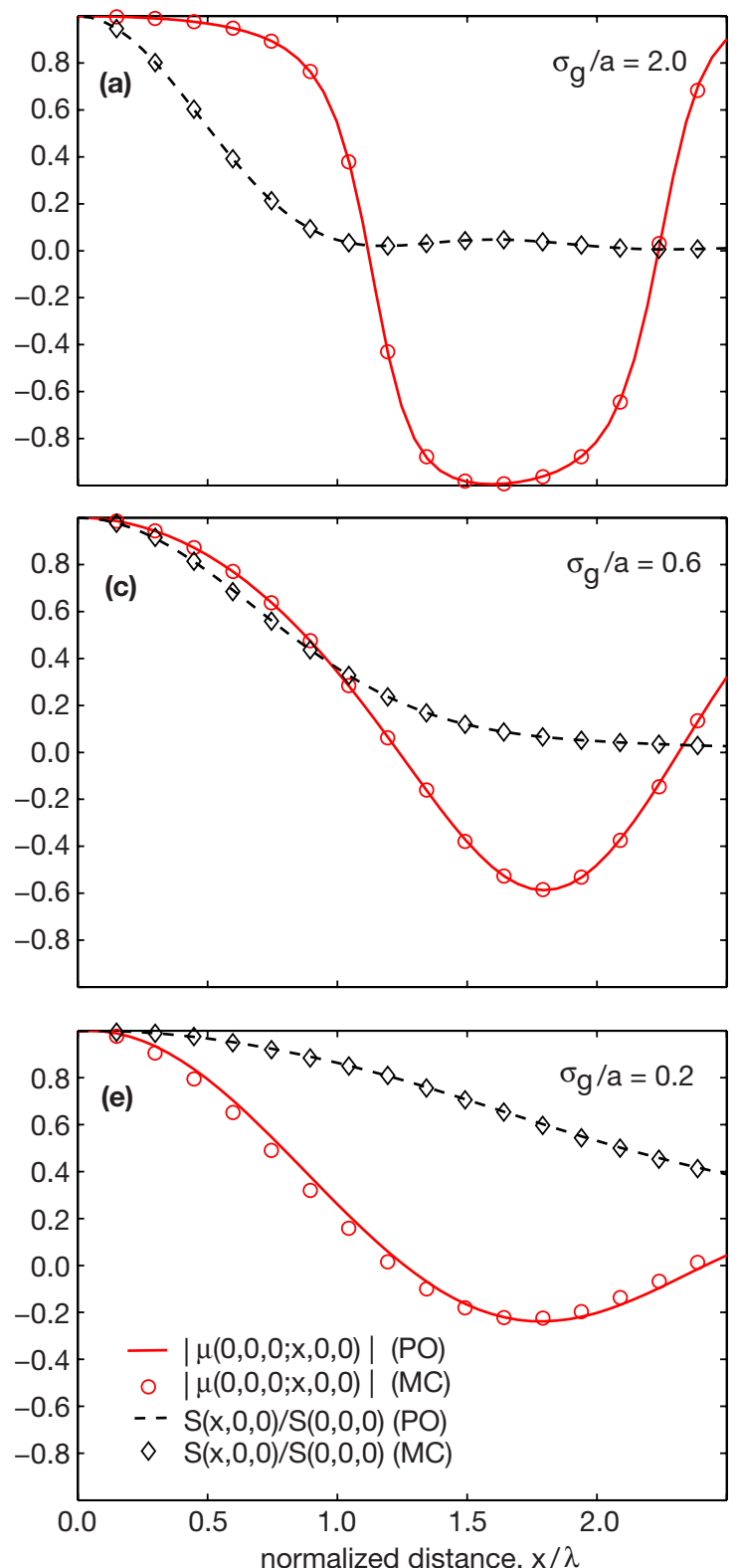

Equation (15) reduces to a (spectral) flux density vector [Eq. (14)] when $\mathbf{r}=\mathbf{r}_{1}=\mathbf{r}_{2}$. The measurement function $M_{P O}$ for the PO cross-spectral density is then given by

$$
M_{P O}\left[W_{P O}\left(\mathbf{r}_{1}, \mathbf{r}_{2}\right)\right]=\left[\mathbf{F}_{c}\left(\mathbf{r}_{1}, \mathbf{r}_{2}\right)\right]_{P O} \cdot \hat{\mathbf{n}}_{D}
$$

where $\hat{\mathbf{n}}_{D}$ is the normal to the detector surface. For nonparaxial fields, it is the quantity defined by Eq. (16) that must be compared with the MC results. For paraxial fields, application of the measurement function is not necessary, as the propagation directions are effectively parallel to the optical axis. When $\hat{\mathbf{n}}_{D}=\hat{\mathbf{z}}$, application of the measurement function results in a difference expression involving two terms, the partial derivative of the crossspectral density with respect to $z_{2}$ and the partial derivative of the cross-spectral density with respect to $z_{1}$. To compute these derivatives numerically, the cross-spectral
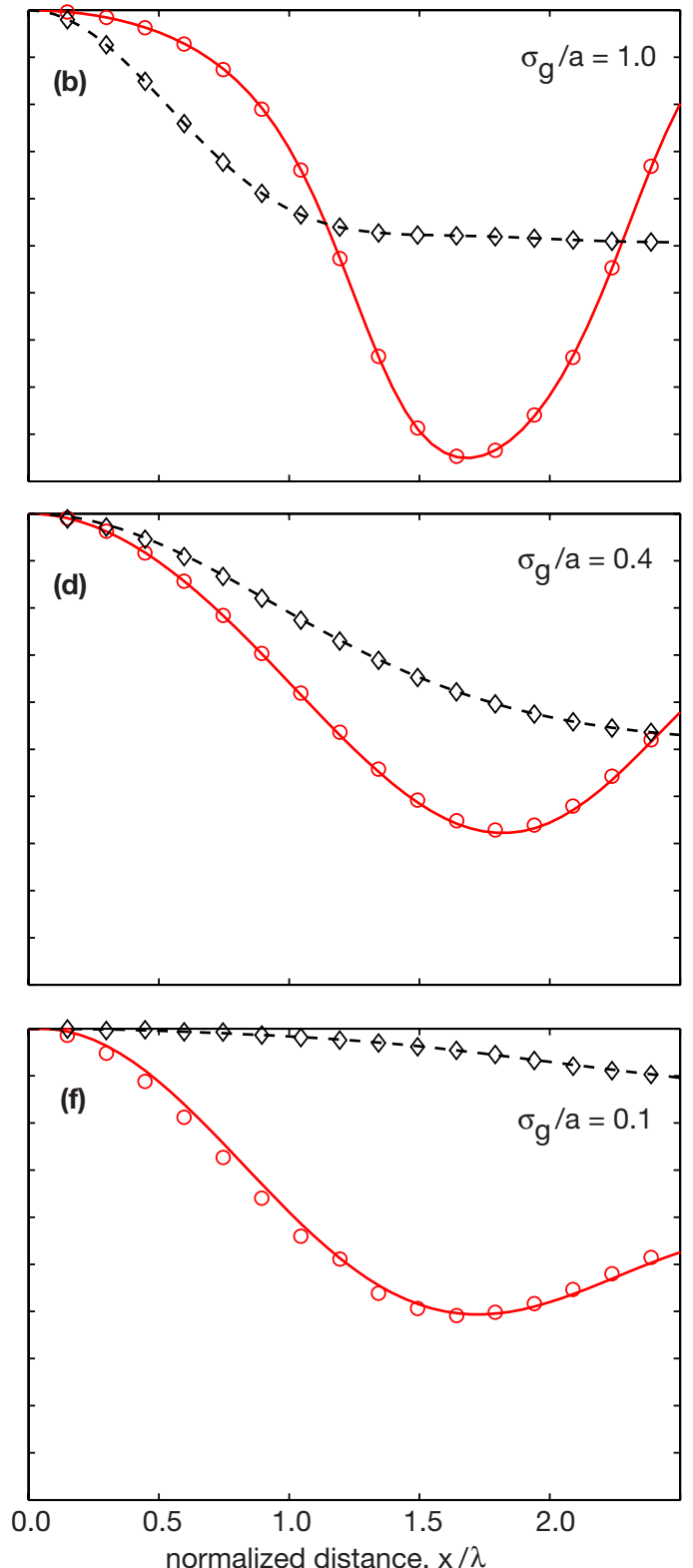

Fig. 6. (Color online) Real part of spectral degree of coherence $\mu(0,0,0 ; x, 0,0)$ and normalized spectral density $S(x, 0,0) / S(0,0,0)$ for various values of $\sigma_{g} / a$ : (a) $\sigma_{g} / a=2.0$, (b) $\sigma_{g} / a=1.0$, (c) $\sigma_{g} / a=0.6$, (d) $\sigma_{g} / a=0.4$, (e) $\sigma_{g} / a=0.2$, (f) $\sigma_{g} / a=0.1$. MC and PO results are for propagation of 65,536 field realizations. 
density must be calculated at two nearby planes (a small distance $\Delta z$ apart) located symmetrically with respect to the focus. This necessarily involves the use of two different Green's functions for propagation of the field realizations to the two planes, respectively.

\section{RESULTS}

Here we present the results of propagating the source cross-spectral density to the focal plane via physical optics (PO) means and by MC ray trace. For the PO calculations, the individual field realizations are propagated according to Eqs. (3) and (8) and the cross-spectral density matrix calculated subsequently. No differences are observed for the alternative approach using Eq. (13). For the MC calculations, Eq. (13) is used with the ray-trace- derived Green's function. The focusing configuration was $\mathrm{f} / 1$, as before. Results are summarized in terms of the spectral density and spectral degree of coherence for varying degrees of coherence within the pupil.

\section{A. Focal Plane Behavior}

Within the focal plane, we use

$$
s=\sqrt{f^{2}+\left(x-x^{\prime}\right)^{2}}
$$

and the observed fields are characterized in terms of the normalized spectral density $S(x, 0,0) / S(0,0,0)$ and spectral degree of coherence $\mu(0,0,0 ; x, 0,0)$. Results for propagating the source cross-spectral density via the $\mathrm{PO}$ and MC Green's function methodology are shown in Fig. 6 . We note that for the PO computations, the standard bi-
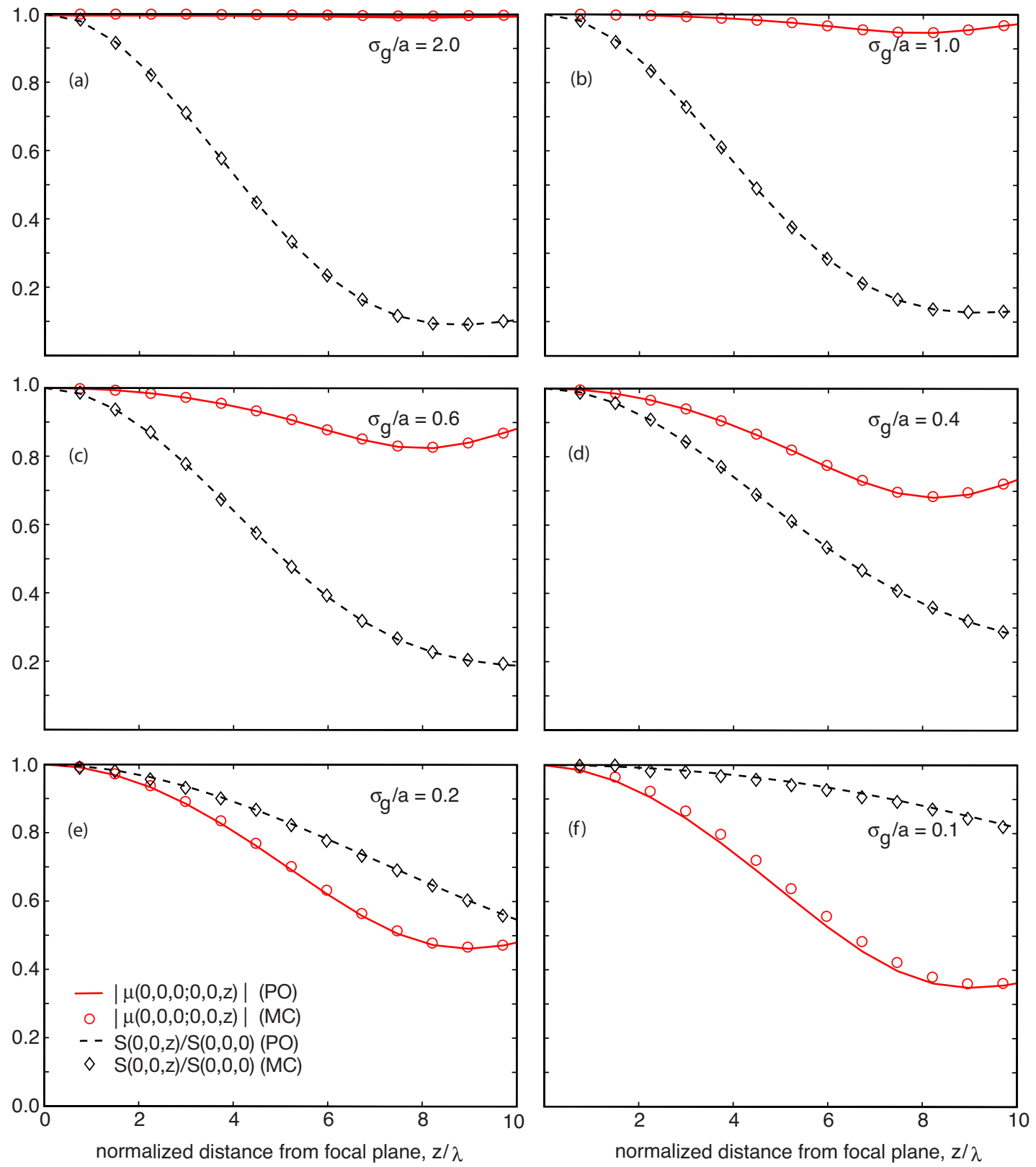

Fig. 7. (Color online) Spectral degree of coherence $\mu(0,0,0 ; 0,0, z)$ and normalized spectral density $S(0,0, z) / S(0,0,0)$ for various values of $\sigma_{g} / a$ : (a) $\sigma_{g} / a=2.0$, (b) $\sigma_{g} / a=1.0$, (c) $\sigma_{g} / a=0.6$, (d) $\sigma_{g} / a=0.4$, (e) $\sigma_{g} / a=0.2$, (f) $\sigma_{g} / a=0.1$. MC and PO results are for propagation of 65,536 field realizations. 
nomial approximation of the expression in Eq. (17) was not used because it is inaccurate for the $f / 1$ configuration. These results are to be compared with Fig. 7 of Fischer and Visser [24], who numerically computed the PO results for the corresponding three-dimensional problem. While the dimensionality of the problem treated herein is different from that of Fischer and Visser, the behaviors are qualitatively the same. Good correspondence is shown between the PO and MC propagation.

\section{B. Axial Behavior}

To evaluate the behavior along the axis near the focus, we use

$$
s=\sqrt{(f-z)^{2}+x^{\prime 2}}
$$

and the observed fields are characterized in terms of the normalized spectral density $S(0,0, z) / S(0,0,0)$ and spectral degree of coherence $\mu(0,0,0 ; 0,0, z)$. Results are summarized in Fig. 7. These results are to be compared with Fig. 5 of Fischer and Visser [24]. Once again, there is very good agreement between the PO and MC predictions.

\section{Application of Measurement Function}

The PO results displayed in Figs. 6 and 7 include a correction due to the measurement function. The magnitude of this correction can be seen if we compare the native $\mathrm{PO}$ results with the corrected PO results. In Figs. 8(a) and
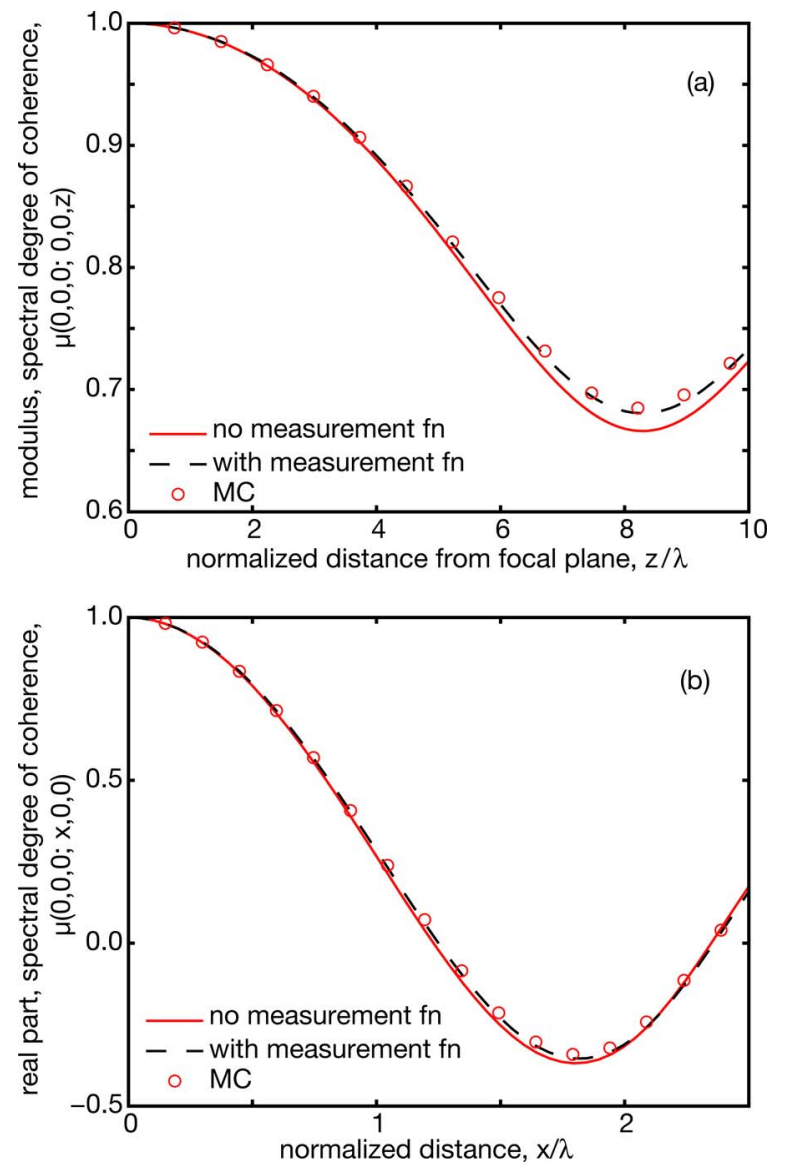

Fig. 8. (Color online) Comparison of the native and corrected PO results for the transverse (a) and axial (b) spectral degrees of coherence for the case $\sigma_{g} / a=0.4$. 8(b), we have repeated the results of Figs. 6(d) and 7(d) for the transverse and axial spectral degrees of coherence, respectively, for the case $\sigma_{g} / a=0.4$. In addition, in both figures we have also plotted the native PO results (before the application of the measurement function). It is seen that application of the measurement results in a noticeable correction to the spectral degree of coherence. Furthermore, this correction will increase as the numerical aperture (NA) of the system is increased (here it is 0.45 ) or the transverse coherence length of the incident illumination is decreased. It should be noted that, while not illustrated here, application of the measurement function also has an effect on the spectral density. It results in a cosine weighting of the spectral density, but this weighting is not apparent over the limited plot range of Figs. 6 and 7 . The correction to the spectral degree of coherence is probably greater because the spectral degree of coherence involves the ratio of two corrected quantities.

\section{Convergence Issues}

By the Green's function formalism we have adopted herein, the issue of convergence of the field statistics has been simplified somewhat [16]. Previously, the convergence of the statistics of the observation plane fields depended on the number of rays per source field realization and the number of realizations. With the current formalism, the number of rays traced is of consequence only for estimation of the Green's function; the number of source realizations is a separate issue altogether.

Convergence of the estimate of the Green's matrix is explored through evaluation of its local standard deviation. For a specified total number of rays traced, the Green's matrix is estimated nine separate times, in each instance with a different initial random number seed. For each entry in the Green's matrix we then compute the standard deviation across all nine estimates. These standard deviations are computed with the formula

$$
\sigma_{i j}^{2}=\frac{1}{8} \sum_{k=1}^{9}\left[\left|G_{i j}^{(k)}\right|-\left|\bar{G}_{i j}\right|\right]^{2},
$$

where $i$ is the index on the detector pixel, $1 \leqslant i \leqslant N ; j$ is the index on the source pixel, $1 \leqslant j \leqslant N^{\prime}$; and $\bar{G}_{i j}$ is the mean Green's function computed over the nine estimates. Figure 9 shows the resulting distributions of these standard deviations for various numbers of traced rays. This figure illustrates that as the number of traced rays increases, the distribution becomes narrower and the mode shifts downward. For each distribution an average is computed; results are shown in Fig. 10. Also shown in this figure is the $1 / \sqrt{N_{r}}$ asymptote, where $N_{r}$ is the number of rays traced. These results suggest that the fidelity of the Green's matrix can be established through inspection of the global mean of the local standard deviations.

We further explore the effect of the number of rays traced and the number of field realizations propagated. This evaluation is in the context of the effect on the spectral density. Results are compared with those computed using PO. As a representative example we choose the partial coherence case of $\sigma_{g} / a=0.6$. Figure 11 is a display of the PO-MC residuals for the focal plane spectral density computed as follows: 


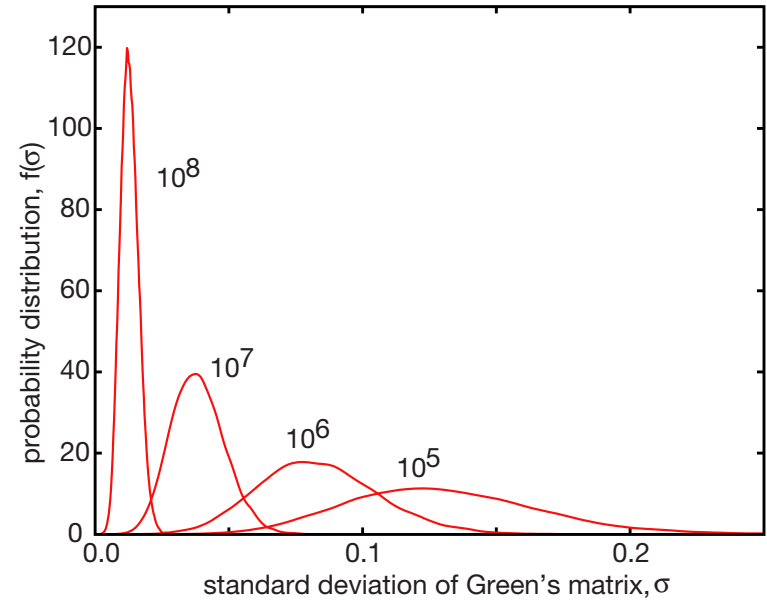

Fig. 9. (Color online) Distribution of standard deviations in Green's matrix for various numbers of traced rays.

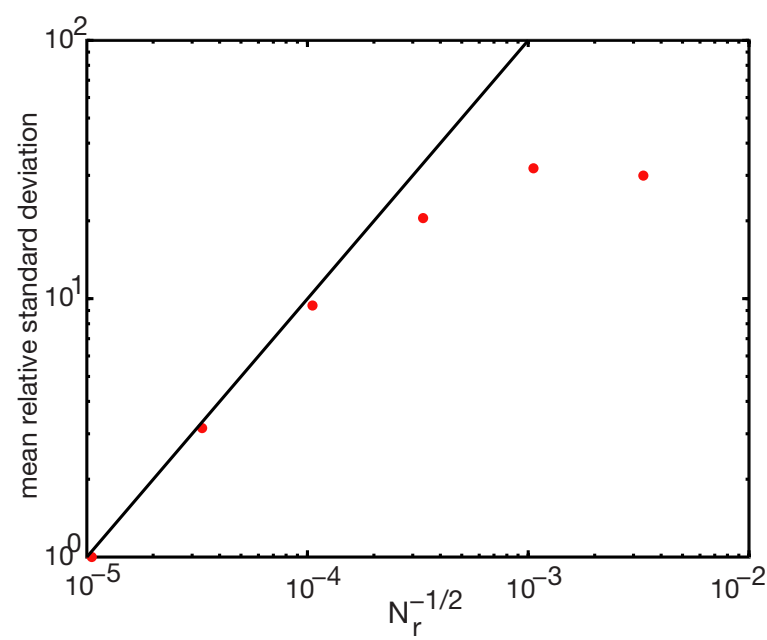

Fig. 10. (Color online) Mean relative standard deviation as a function of number of rays propagated. Also shown is the $1 / \sqrt{N_{r}}$ asymptote.

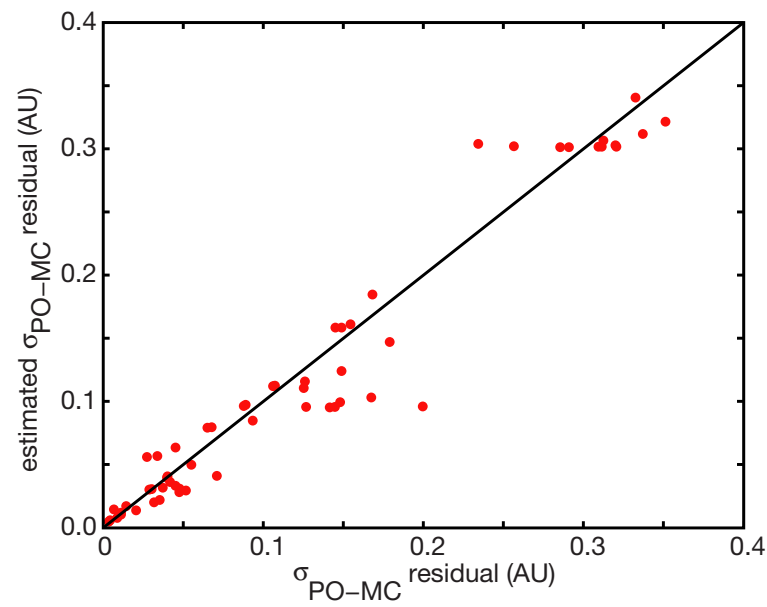

Fig. 11. (Color online) PO-MC residuals versus model that is a function of number of rays traced and number of field realizations propagated. Solid line is the identity.

$$
\sigma_{P O-M C}^{2}=\frac{1}{N-1} \sum_{i=1}^{N}\left[\frac{S_{P O}\left(x_{i}, 0,0\right)}{S_{P O}(0,0,0)}-\frac{S_{M C}\left(x_{i}, 0,0\right)}{S_{M C}(0,0,0)}\right]^{2},
$$

where the summation is over the $N$ detector elements. These residuals are plotted against a fit model,

$$
\hat{\sigma}_{P O-M C}^{2}=\left(\frac{30.1}{\sqrt{N_{r}}}\right)^{2}+\left(\frac{0.895}{\sqrt{N_{f}}}\right)^{2},
$$

where $N_{r}$ and $N_{f}$, are respectively, the number of rays traced and the number of field realizations propagated. This model explains the residuals well and demonstrates the independence of the number of rays and the number of field realizations (due to the additivity of the variances). Not surprisingly, errors in the Green's function have a greater influence on the final result than the number of fields propagated.

\section{DISCUSSION AND CONCLUSIONS}

We have presented some recent results of our efforts toward the development of a Monte Carlo formalism for propagating fully stochastic sources through free space and in the presence of diffracting structures. Use of the Green's function formalism has some obvious benefits, aside from the commonly recognized features of such MC calculations (simplicity, ease of treating complex boundary conditions, etc.). In a previous paper [16], we addressed the issue of convergence of the estimates for propagation of an ensemble of field realizations. We showed that based on the first-order statistics of the detector plane field, one could distinguish between convergence of the field estimates from the numerical propagation effects. With the Green's function formalism, one explicitly separates the issue of convergence of the $\mathrm{MC}$ algorithm from the statistical behavior of the detector plane fields. Another benefit of the MC Green's function concept (and the MC ray trace concept in general) is that there is no spatial quantization aside from that of the source and detector planes. As a result, no explicit consideration need be given to adequate sampling of the steeply sloped wavefronts as seen in high NA configurations.

We have chosen a problem for which the $\mathrm{MC}$ and $\mathrm{PO}$ calculations are relatively straightforward. For such a case, the MC calculations can be verified with direct numerical integration of the $\mathrm{PO}$ integrals. The end result is validation of the MC method of propagating partially coherent fields. The real utility of the MC Green's function concept, however, lies in more interesting situations (high NA imaging systems, three-dimensional problems, strongly scattering media, etc.). These problems are ones for which PO calculations become difficult or impossible. Nevertheless, it is these problems that lend themselves to treatment using the MC Green's function approach. Having established the validity of this new approach, the substantiating PO calculations become unnecessary. The formalism that we have developed obviates some of the shortcomings of PO approaches, is straightforward to implement numerically, and lends itself to parallel computation. For complex scattering media, the effective- 
medium of conventional MC approaches must be supplanted by a structured model such as the phase screen paradigm [1] used for propagation through atmospheric turbulence. Our current MC approach is entirely capable of describing diffraction from such structures, and the copula algorithm is well suited for generating realizations of structured stochastic media. This ongoing research will be the subject of future publications.

The interaction of light with matter is fundamental to the understanding of our physical surroundings; electromagnetic energy is an observable that carries with it the fingerprint of its interaction with matter. As a result, the features of this light are the means by which we understand the universe around us. Yet the propagation of light within highly scattering media is not well understood. A thorough understanding of this phenomenon is therefore requisite for any endeavor that attempts to characterize a physical medium, whether biological tissue, earth's atmosphere, or interstellar space. The efforts described in this paper, which represent an attempt to directly link the theory of light as a coherent wave phenomenon with radiometric methods as embodied in MC studies of light propagation, are aimed at developing a formalism for studying such a wide ranging class of problems that cannot be treated with either one approach or the other by itself.

\section{APPENDIX A}

Consider the phasor summation

$$
U=\frac{1}{\sqrt{N}} \sum_{k=1}^{N} \exp \left(i \alpha_{k}\right) .
$$

It is easily demonstrated [22] that if the $\alpha_{k}$ is independent and each is uniformly distributed on the interval $(-\pi, \pi)$, then $U$ is a circular complex Gaussian random variable of zero mean and unit variance. This is the requirement, for example, for a laser speckle pattern to be considered "fully developed." Thus it would seem that the requirement on the phase distribution (of the $\alpha_{k}$ ) is fairly stringent. In fact, it is not. It is sufficient that the phase distribution effectively span the fundamental interval. For example, consider the phase distribution illustrated in

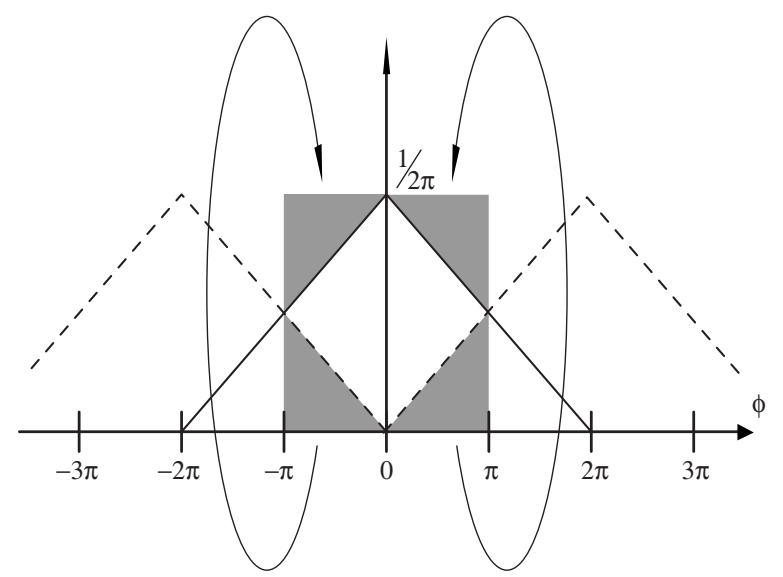

Fig. 12. Illustration of phase wrapping from adjacent Riemann sheets into the fundamental interval.
Fig. 12. The phases outside the fundamental interval are wrapped into the interval $(-\pi, \pi)$, thus producing a phase that is effectively uniformly distributed. Another example might be the case in which the phases have a zero-mean Gaussian distribution. It is straightforward to show that for the standard deviation exceeding approximately $\pi$, the phase is effectively uniformly distributed and thus the phasor sum [Eq. (A1)] has circular complex Gaussian statistics.

\section{ACKNOWLEDGMENT}

Scott Prahl recognizes partial support from National Institutes of Health (NIH), grant 1R21DE016758-01A2.

\section{REFERENCES}

1. L. C. Andrews and R. L. Phillips, Laser Beam Propagation through Random Media (SPIE Press, 1998).

2. G. Gbur and E. Wolf, "Spreading of partially coherent beams in random media," J. Opt. Soc. Am. A 19, 1592-1598 (2002).

3. G. Yao and L. V. Wang, "Monte Carlo simulation of an optical coherence tomography signal in homogeneous turbid media," Phys. Med. Biol. 44, 2307-2320 (1999).

4. Q. Lu, X. Gan, M. Gu, and Q. Luo, "Monte Carlo modeling of optical coherence tomography imaging through turbid media," Appl. Opt. 43, 1628-1637 (2004).

5. C. Mujat and A. Dogariu, "Statistics of partially coherent beams: a numerical analysis," J. Opt. Soc. Am. A 21, 1000-1003 (2004).

6. F. Mayinger and O. Feldmann, eds., Optical Measurements: Techniques and Applications (Springer-Verlag, 2001).

7. J. Pawley, ed., Handbook of Biological Confocal Microscopy (Springer, 1995).

8. M. Keijzer, S. L. Jacques, S. A. Prahl, and A. J. Welch, "Light distributions in artery tissue: Monte Carlo simulations for finite-diameter laser beams," Lasers Surg. Med. 9, 148-154 (1989).

9. L. Wang, S. L. Jacques, and L. Zheng, "MCML-Monte Carlo modeling of light transport in multi-layered tissues," Comput. Methods Programs Biomed. 47, 131-146 (1995).

10. S. Bartel and A. H. Hielscher, "Monte Carlo simulations of the diffuse backscattering Mueller matrix for highly scattering media," Appl. Opt. 39, 1580-1588 (2000).

11. L. Mandel and E. Wolf, Optical Coherence and Quantum Optics, (Cambridge U. Press, 1999).

12. A. M. Zysk, P. S. Carney, and J. C. Schotland, "Eikonal method for calculation of coherence functions," Phys. Rev. Lett. 95, 043904 (2005).

13. J. C. Petruccelli and M. A. Alonso, "Ray-based propagation of the cross-spectral density," J. Opt. Soc. Am. A 25, 1395-1405 (2008).

14. N. G. Douglas, A. R. Jones, and F. J. van Hoesel, "Raybased simulation of an optical interferometer," J. Opt. Soc. Am. A 12, 124-131 (1995).

15. F. Riechert, F. Dürr, U. Rohlfing, and U. Lemmer, "Raybased simulation of the propagation of light with different degrees of coherence through complex optical systems," Appl. Opt. 48, 1527-1534 (2009).

16. D. G. Fischer, S. A. Prahl, and D. D. Duncan, "Monte Carlo modeling of spatial coherence: free-space diffraction," J. Opt. Soc. Am. A 25, 2571-2581 (2008).

17. N. Metropolis and S. Ulam, "The Monte Carlo method," J. Am. Stat. Assoc. 44, 335-341 (1949).

18. J. J. Stamnes, Waves in Focal Regions: Propagation, Diffraction, and Focusing of Light, Sound, and Water Waves (Adam Hilger, 1986).

19. R. B. Nelson, An Introduction to Copulas (Springer-Verlag, 1999). 
20. D. D. Duncan and S. J. Kirkpatrick, "The copula: a tool for simulating dynamic speckle," J. Opt. Soc. Am. A 25 231-237 (2008).

21. A. Papoulis and S. U. Pillai, Probability, Random Variables, and Stochastic Processes, 4th ed. (McGraw-Hill, 2002).

22. W. Goodman, "Statistical properties of laser speckle patterns," in Laser Speckle and Related Phenomena, J. C. Dainty, ed. (Springer-Verlag, 1975), pp. 9-75.

23. J. W. Goodman, Statistical Optics (Wiley, 1985).

24. D. G. Fischer and T. D. Visser, "Spatial correlation properties of focused partially coherent light," J. Opt. Soc. Am. A 21, 2097-2102 (2004). 